\title{
Gastkommentar zum Beitrag „Frühgeburtenrate in 6 Perinatalzentren in Baden-Württemberg - Potenzial zur Reduktion der Frühgeborenenzahl“‘: Reduktion der Frühgeburtenrate durch mehr Transparenz. Was ist der geburtshilfliche Aspekt?
}

\author{
Autor \\ Irene Hösli \\ Institut \\ Klinik für Geburtshilfe und Schwangerschaftsmedizin, \\ Frauenklinik Universitätsspital Basel, Schweiz \\ Bibliografie \\ DOI https://doi.org/10.1055/a-0744-1261 \\ Z Geburtsh Neonatol 2018; 222: 207 \\ (c) Georg Thieme Verlag KG Stuttgart · New York \\ ISSN 0948-2393
}

\author{
Korrespondenzadresse \\ Prof. Dr. Irene Hösli \\ Geburtshilfe und Schwangerschaftsmedizin \\ Abteilung Geburtshilfe und Schwangerschaftsmedizin \\ Frauenklinik, Universitätsspital Basel \\ Spitalstrasse 21 \\ 4031 Basel \\ Schweiz \\ irene.hoesli@usb.ch
}

Die vorliegende Beobachtungsstudie «Frühgeburtenrate in 6 Perinatalzentren in Baden-Württemberg-Potenzial zur Reduktion der Frühgeburtenzahl» nimmt Bezug auf die sehr unterschiedliche Frühgeburtenrate in Perinatalzentren. Zwischen dem Zentrum mit der höchsten und dem mit der tiefsten Frühgeburtenrate bestand mehr als $10 \%$ Differenz. Je tiefer die Frühgeburtenrate desto tiefer lag auch die neonatale Morbidität. Auch wenn die demografischen Daten und fetalen Charakteristika der einzelnen Zentren nicht bekannt sind und sicher variieren, muss doch angenommen werden, dass auch das unterschiedliche geburtshilfliche Management eine grosse Rolle gespielt hat. Dies spiegelt sich besonders in der Subpopulation: Frühgeburt nach vorzeitigem frühem spontanem Blasensprung (PPROM) wider. Das Risiko für eine Frühgeburt nach PPROM ist mit über $70 \%$ insgesamt sehr hoch. Der Unterschied der Frühgeburtenrate betrug zwischen dem Zentrum mit der höchsten und dem mit der tiefsten Frühgeburtenrate mehr als $35 \%$. Die Betreuung beim PPROM gehört in der Geburtshilfe zu einem sehr kontrovers diskutierten Thema und variiert zwischen aggressivem raschen Entbinden und konservativ expektativem Vorgehen. Auch die Empfehlungen verschiedener internationaler Guidelines zur Diagnostik und zum Management des PPROM reflektieren diese Heterogenität [1]. Die beiden anderen Einschlusskriterien verkürzte Cervix unter $25 \mathrm{~mm}$ und vorzeitige Wehen waren wesentlich häufiger, führten aber nur in 20-26\% zu einer Frühgeburt. Aber auch hier zeigten sich Unterschiede von über $10 \%$ zwischen dem Zentrum mit der höchsten und dem mit der tiefsten Frühgeburtenrate.

Wie können wir die Rate an Frühgeburten reduzieren? Es braucht sicherlich Struktur- und Personalvorgaben, um die Qualität der Frühgeburtenversorgung zu optimieren. Es braucht aber auch evidenzbasierte präventive und therapeutische Massnahmen, um die Frühgeburten zu vermeiden bzw. optimal auf die Geburt vorzubereiten.
Aktuell wird an der DGGG Leitlinie Frühgeburt interdisziplinär gearbeitet. Ausserdem braucht es Aufklärung, den Sektor public health und zuletzt weitere Forschung, um den zugrundeliegenden Mechanismus besser verstehen und therapeutische Interventionen anpassen zu können. Die auch international bestehenden unterschiedlichen Raten an Frühgeburten in Ländern mit hohem Einkommen sind in zwei Drittel nicht biologisch zu erklären [2].

Die Arbeit leitet eine klare Forderung ab: anstelle einer weiteren Mindestmengenerhöhung an Frühgeburten die zentrumsspezifische Frühgeburtenrate zu veröffentlichen und damit transparent zu machen. Es sollte kein Anreiz sein, möglichst viele Frühgeburten zu haben, sondern möglichst viele zu vermeiden und die neonatale Morbidität und Mortalität zu senken.

Interessenkonflikt

Die Autoren geben an, dass kein Interessenkonflikt besteht.

Literatur

[1] Tsakiridis I, Mamopoulos A, Chalkia-Prapa EM et al. Preterm Premature Rupture of Membranes: A Review of 3 National Guidelines. Obstet Gynecol Surv 2018; 73: 368-375. doi:10.1097/OGX.0000000000000567

[2] Ferrero DM, Larson J, Jacobsson B et al. Cross-Country Individual Participant Analysis of 4.1 Million Singleton Births in 5 Countries with Very High Human Development Index Confirms Known Associations but Provides No Biologic Explanation for 2/3 of All Preterm Births. PLoS One. 2016; 11: e0162506. doi:10.1371/journal.pone.0162506. eCollection 2016 